\title{
Análise de Duas Tecnologias Comercialmente Disponíveis para o Controle do Sistema de Condicionamento de Ar da UFMT: Um Estudo de Caso
}

\section{Analysis of Two Commercially Available Technologies for Control of UFMT Air Conditioning System: A Case Study}

\author{
${ }^{1}$ Bruno Bombazaro Menegotto, ${ }^{2}$ Roberto Perillo Barbosa da Silva, ${ }^{3}$ Rodolfo Quadros, ${ }^{4}$ Danilo Ferreira de Souza \\ 1,3 Engenheiro Eletricista pela Universidade Federal de Mato Grosso - UFMT ( bbombazaro@outlook.com) \\ 2,4 Professores mestres no Departamento de Engenharia Elétrica - UFMT ( danilo@ufmt.br)
}

Recebido em: Novembro/2016 Aceito em : dezembro de 2016 Publicado: junho de 2016

\begin{abstract}
Resumo: Este trabalho apresenta um estudo a respeito de um sistema de condicionadores de ar utilizado na Universidade Federal de Mato Grosso-UFMT, realizando análises comparativas em relação a proposta de implantação de um sistema de controle destes equipamentos. Para tanto, foi feita a medição de energia elétrica $(\mathrm{kWh})$ consumida por 05 (cinco) condicionadores de ar nas salas da Faculdade de Medicina da UFMT, durante o período de uma semana, identificando o perfil de funcionamento. Foi constatado desperdício de energia elétrica da ordem de 11,9\%. Identificou-se assim, um potencial para economia deste insumo, onde é possível realizar controles relacionados aos condicionadores de ar, como controle do tempo para utilização e da temperatura interna dos ambientes, uma vez que estas variáveis ocasionam um impacto direto no consumo de energia elétrica em sistemas de condicionadores de ar. Foram propostas duas estratégias de funcionamento para o controle dos equipamentos. Com base no estudo de caso e nos investimentos levantados, estimaram-se os tempos de retorno de 22 meses para a primeira estratégia e de 27 meses para a segunda estratégia, onde ambas as estratégias consideram um cenário em que o sistema é empregado em 5 (cinco) salas de aula. Também foram criados cenários e realizadas análises para obter o valor do investimento inicial e do tempo de retorno simples, onde o sistema de controle, utilizando a primeira e a segunda estratégias, é empregado em cenários com 10, 15 e 20 salas de aula.
\end{abstract}

Palavras-chave: Condicionador de Ar. Sistema de Controle. Uso Racional de Energia Elétrica.

\begin{abstract}
This work presents a study about a system of air-conditioners used in the Federal University of Mato Grosso, carrying out comparative analyzes in relation to the proposal for implementation of a system of control of such equipment. For both, was performed measurement of electric energy (kWh) consumed by 05 (five) airconditioners in the rooms of the Faculty of Medicine of UFMT, during a period of one week, identifying the profile of operation. It was observed a waste of electricity of the order of $11,9 \%$. It was identified as a potential for the economy of this input, where it is possible to perform controls related to air conditioners, as time control for use and the internal temperature of the environments, since these variables, provoking a direct impact on the consumption of electric energy in systems of air-conditioners. Two strategies have been proposed for the control of the equipment. On the basis of case study and investments raised, we estimated the times of return of 22 months for the first strategy and 27 months for the second strategy, where both strategies consider a scenario in which the system is used in 5 (five) classrooms. Also were created scenarios and analyzes to obtain the value of the initial investment and the return time of simple, where the control system, using the first and second strategies, it is employed in scenarios with 10, 15 and 20 classrooms.
\end{abstract}

Key words: Air Conditioner. Control System. Rational Use of Electrical Power

\section{INTRODUÇÃO}

No Brasil, o consumo perdulário (desperdiçado) de energia elétrica é avaliado em cerca de $20 \%$ do consumo total de energia elétrica do país (SANTOS et al, 2006), tendo, portanto, uma enorme lacuna a ser preenchida com projetos que contemplem a economia de energia elétrica.

Os aspectos do combate ao desperdício têm como principais motivadores (SANTOS et al, 2006): 
- A economia de recursos, devido à possibilidade de postergação dos investimentos em sistemas de geração, transmissão e distribuição de energia;

- Aumento da competitividade dos bens e serviços produzidos;

- A proteção e a melhoria do meio ambiente.

A importância da conscientização e educação do consumidor no combate aos desperdícios é salientada pelos resultados diretos em economia de recursos e benefícios ambientais, provocados pela mudança de hábitos e comportamentos, quanto à correta utilização dos equipamentos, sistemas e processos, cumprindo um papel essencial na formulação de ações voltadas para o uso racional de energia elétrica, apresentando resultados positivos, tanto no aspecto econômico, quanto no impacto ao meio ambiente.

Estudos apontam uma divisão do consumo de eletricidade em edifícios entre as seguintes cargas: $48 \%$ pelo condicionamento de ar, $23 \%$ pela iluminação, $15 \%$ por equipamentos de escritório e $14 \%$ devido às demais cargas, como bombas e elevadores (PROCEL, 2007). Estes dados ressaltam a importância de se considerar um sistema de controle de condicionadores de ar, tema para o qual este trabalho está direcionado, no sentido de realizar uma análise comparativa, sob aspectos financeiros e do uso racional de energia elétrica, do potencial de economia de energia elétrica através da análise de um sistema de controle de condicionadores de ar que utiliza um Interruptor Horário, analisado sob uma estratégia de funcionamento e outro sistema, que utiliza um Controlador Lógico Programável (CLP), o qual foi analisado sob uma segunda estratégia de funcionamento.

A proposta é que este sistema não permita o acionamento dos condicionadores de ar fora dos horários de funcionamento dos ambientes analisados, com o objetivo de reduzir o consumo e possibilitar a utilização da energia elétrica de maneira racional, conforme programação a ser desenvolvida no CLP ou no Interruptor Horário, uma vez que, na configuração atual, os aparelhos podem ser acionados livremente, sem qualquer controle.

Os objetivos deste trabalho são:

- Identificar a curva de operação do sistema de condicionamento de ar em estudo, através da realização de medição do consumo de energia elétrica no Quadro de Distribuição, que alimenta o conjunto de condicionadores de ar, com o objetivo de obter o consumo diário, que corresponda ao perfil de utilização típico dos aparelhos nos ambientes em questão;

- Analisar o potencial de economia de energia elétrica;

- Analisar o tempo de retorno simples do investimento com a economia de energia obtida, frente às diferentes alternativas;

- Propor um sistema que possa ser replicado facilmente nos demais sistemas de condicionamento de ar da Universidade, em seus diversos campi.

\section{MATERIAIS E MÉTODOS}

\section{Local do Estudo}

O local objeto do estudo encontra-se na cidade de Cuiabá, no Câmpus Gabriel Novis Neves - UFMT. O quadro de distribuição, onde foi realizada a medição de energia, está localizado no bloco do Centro de Ciências Biomédicas e Saúde I (CCBS I), sendo este responsável por alimentar os condicionadores de ar pertencentes às 5 salas de aula da Faculdade de Medicina (FM). A disposição das salas de aula e a localização do quadro de distribuição são mostradas na Figura 1. 
Figura 1- Disposição das Salas de Aula Dentro do Bloco CCBS I.

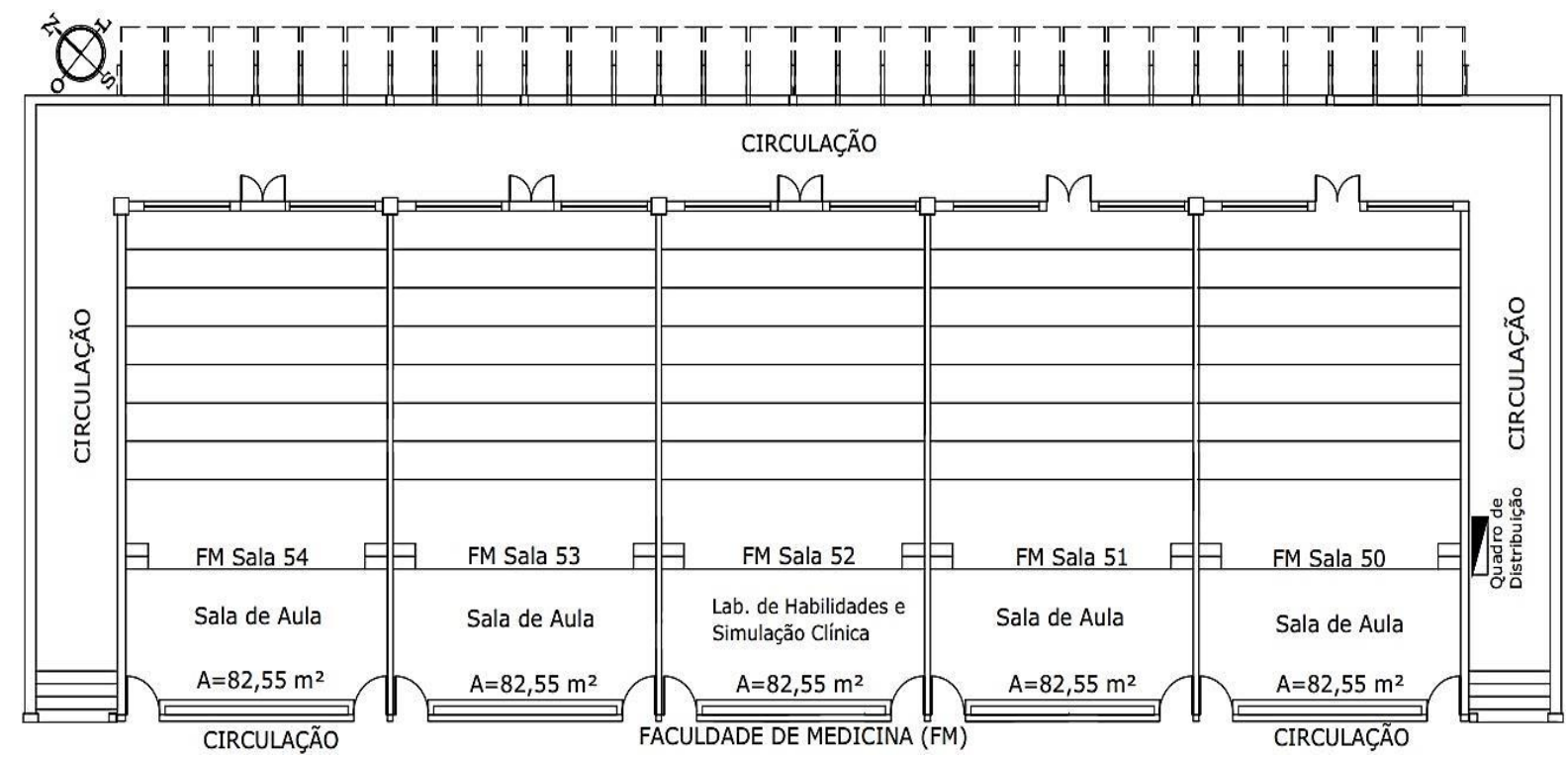

Fonte: Autoria Própria (2015).

As salas de aula possuem condicionadores de ar do tipo janela e do tipo Split. O quadro de distribuição é responsável por alimentar exclusivamente os circuitos terminais de 11 condicionadores de ar, tal que os aparelhos existentes nas salas de aula estão listados na Tabela 1, divididos por tipo e potência nominal.

Tabela 1 - Classificação dos Condicionadores de Ar Existentes nas Salas de Aula.

\begin{tabular}{ccc}
\hline Quantidade & Tipo & Potência (Btu/hora) \\
\hline 07 & split & 48.000 \\
02 & split & 30.000 \\
02 & janela & 30.000 \\
\hline
\end{tabular}

Fonte: Autoria Própria (2015).

\section{Materiais}

\section{Componentes do Sistema de Controle}

O sistema de controle proposto deverá ser composto de:

- Contatora;

- Controlador Lógico Programável ou Interruptor Horário;

- Disjuntor Termomagnético Bipolar;

- Quadro de Distribuição.
Na Figura 2 é apresentado o esquema de ligação do sistema de controle proposto, para o cenário com 5 salas de aula, no qual o Quadro de Controle, onde serão alocados o CLP ou Interruptor Horário (depende da estratégia a ser utilizada), a contatora e os disjuntores, é acionado pelo disjuntor termomagnético tripolar de $100 \mathrm{~A}$, do quadro de distribuição existente. Ou seja, este disjuntor deverá ser retirado do quadro responsável por alimentar os condicionadores de ar e ser inserido no Quadro de Controle, como disjuntor geral. Uma das saídas do CLP ou do Interruptor Horário deverá ser conectada a um dos terminais da bobina da contatora responsável por desligar o quadro de distribuição existente, sendo o conjunto bobina da contatora - CLP/bobina da contatora - Interruptor Horário acionado pelo disjuntor termomagnético bipolar de $10 \mathrm{~A}$.

As ligações entre disjuntor geral, contatos de força da contatora e barramento do Quadro de Distribuição existente (Quadro de Distribuição AR) são feitas com cabo de Cobre isolado PVC $450 / 750 \mathrm{~V}$ de seção igual a \#35 mm². Na Figura 2, A1 - A2 são os terminais da bobina da contatora $\mathrm{C} 1$ e DJ 2x10A representa o disjuntor termomagnético bipolar de 10A. 
Figura 2 - Esquema de Ligação do Sistema de Controle Proposto.

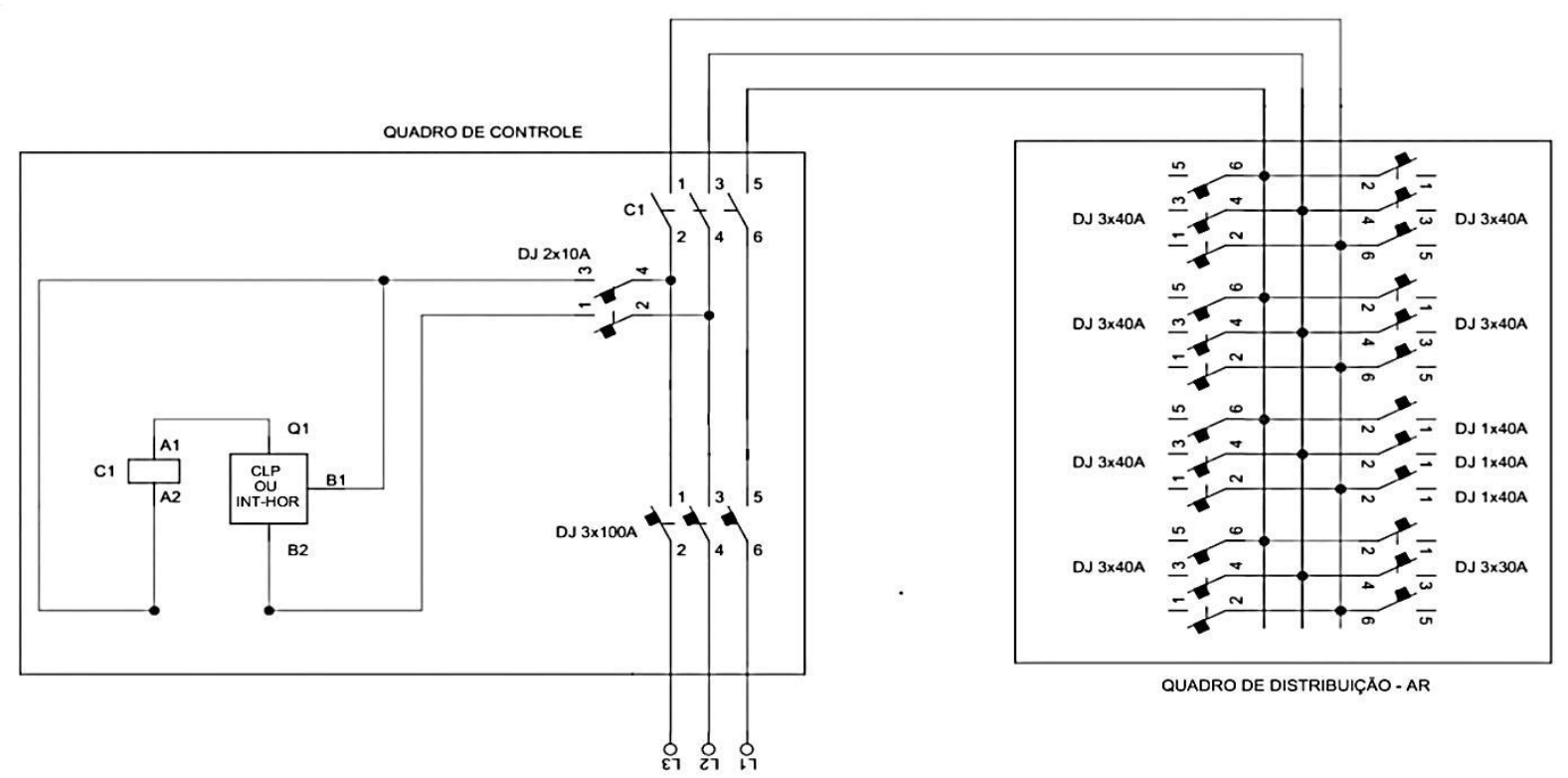

Fonte: Autoria Própria (2015).

\section{Medidor de Energia}

Para a realização da medição de energia elétrica foi utilizado o Medidor Universal de Grandezas (MUG), modelo ELO 2160, o qual foi instalado junto ao quadro de distribuição que alimenta os condicionadores de ar.

A partir dos sinais de entrada de tensão e corrente, o MUG pode disponibilizar, dependendo da ligação utilizada (estrela ou triângulo), os valores de corrente de neutro, corrente elétrica mínima, média e máxima nas fases A, B e C, tensão mínima, média e máxima de linha e de fases, potência ativa por fase e trifásica, potência reativa por fase e trifásica, potência aparente por fase e trifásica, fator de potência por fase e trifásico, energia ativa trifásica e energia reativa trifásica.

A programação do aparelho consiste em pressionar as teclas que controlam as indicações do mostrador de cristal líquido para a visualização das grandezas medidas.

\section{MÉTODOS}

\section{Medição}

Para a análise do consumo de energia elétrica nos ambientes escolhidos, foi instalado o medidor de energia elétrica (MUG) no quadro de distribuição responsável pela alimentação dos condicionadores de ar. A medição foi realizada no período de 4 a 11 de novembro de 2014, ou seja, 07 (sete) dias de medição, o que representa uma semana típica de aulas na universidade.

Os parâmetros medidos foram retirados da memória de massa do medidor, com o auxílio do software do fabricante, sendo exportados para uma planilha eletrônica.

Na Figura 3 são mostrados os clamps de corrente, as garras para amostra da tensão e o MUG (canto superior direito), instalados no quadro de distribuição. 
Figura 3 - Clamps de Corrente e Garras de Tensão Instalados no Quadro de Distribuição para Medição.

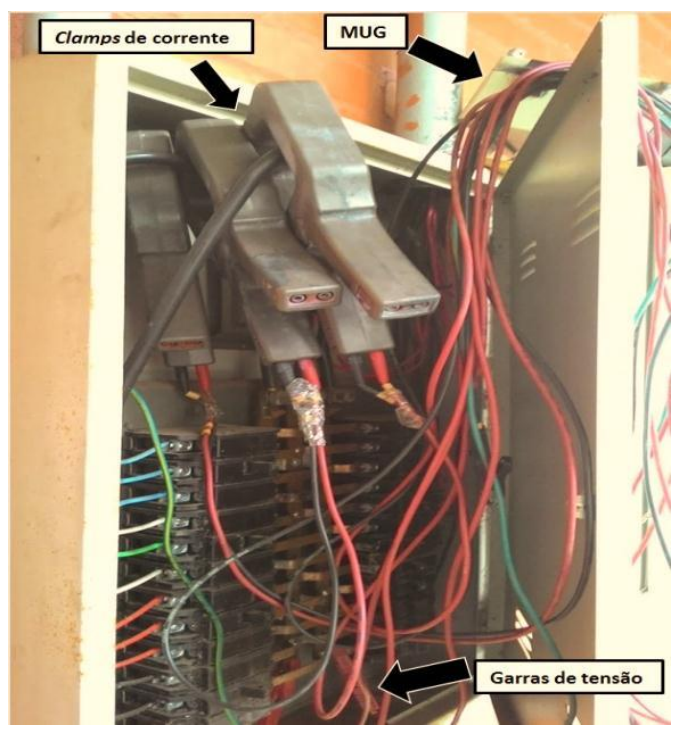

Fonte: Autoria Própria (2014).

\section{Estratégias para o Controle dos Condicionadores de Ar}

Foram adicionados 5 minutos de tolerância antes do início das aulas, para que a análise do comportamento do sistema de controle estivesse mais próxima da realidade de utilização das salas de aula. Ou seja, levouse em consideração casos em que a sala de aula é utilizada antes do início da aula para preparação do material de apoio, como data show. O sistema possui flexibilidade em sua configuração, portanto, é possível adicionar um tempo diferente, como, por exemplo, 10 minutos de tolerância. Não foram adicionados 5 minutos de tolerância após o horário das aulas, pois, devido à inércia térmica e a redução da quantidade de pessoas que permanecem dentro do ambiente, a temperatura do ambiente demora um tempo maior para sair da zona de conforto térmico, do que o tempo necessário para que sejam encerradas todas as atividades dentro da sala de aula. Entretanto, devido à flexibilidade do sistema, é possível adicionar, quando se mostrar adequado, esta tolerância no tempo de funcionamento após o horário das aulas.
De acordo com o exposto acima, a primeira estratégia de funcionamento para o sistema de controle utiliza um Interruptor Horário com uma programação que permite a utilização dos condicionadores de ar de acordo com o horário normal das aulas, ou seja: das 07h25min às 11h30min, das $13 \mathrm{~h} 25 \mathrm{~min}$ às $17 \mathrm{~h} 30 \mathrm{~min}$ e das $17 \mathrm{~h} 5 \mathrm{~min}$ às $22 \mathrm{~h} 00 \mathrm{~min}$.

A segunda estratégia de funcionamento do sistema de controle utiliza um CLP operando como um Interruptor Horário, permitindo a utilização dos condicionadores de ar nos mesmos horários e com os mesmos objetivos da primeira estratégia. Esta estratégia é semelhante à primeira em termos de funcionamento, mas permite a expansão do sistema de controle, onde se pode realizar o controle do uso dos condicionadores de ar por sala, além de possibilitar o emprego de mais dispositivos do tipo sensores para auxiliar no controle.

\section{RESULTADOS}

Os resultados são apresentados para cada estratégia de controle proposta, onde todas se fundamentam no objetivo geral deste trabalho, que é analisar duas tecnologias comercialmente disponíveis que possam compor um sistema de controle dos condicionadores de ar, visando contribuir para a redução do consumo de energia elétrica. Para tanto, cada estratégia foi analisada sob a ótica do potencial de economia de energia elétrica e dos custos envolvidos para sua implantação, mediante o cenário de um conjunto de 5 salas de aula, onde existem os mesmos condicionadores de ar listados na Tabela 1.

\section{Análise do Consumo de Energia Elétrica das Salas de Aula sem Automação}

O cenário atual do uso de energia elétrica no câmpus da UFMT em Cuiabá é ilustrado pela Figura 4, mostrando o comportamento da demanda, em $\mathrm{kW}$, de um 
Menegotto et.al., Análise de Duas Tecnologias Comercialmente Disponíveis para o Controle do Sistema de Condicionamento de Ar da UFMT: Um Estudo de Caso. E\&S - Engineering and Science, (2016), 5:1.

dia típico de atividades no câmpus (02/10/2014).

Figura 4 - Demanda, em kW, de um dia Típico de Atividades no Câmpus da UFMT em Cuiabá.

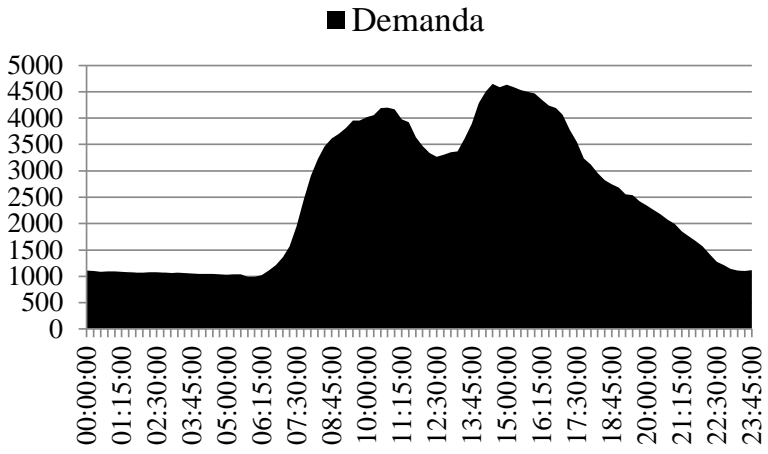

Fonte: Autoria Própria (2015), com base nos dados medidos por Reis (2015).

Este perfil de demanda se assemelha à medição feita, durante uma semana (4 à 11 de novembro de 2014), no quadro de distribuição que alimenta 11 condicionadores de ar de 5 salas de aula (estudo de caso no qual se baseia este trabalho), na Faculdade de Medicina. A Figura 5 também mostra uma queda acentuada, no horário de almoço dos dias 05 , 06, 07, 10 e 11, no consumo de energia ativa $(\mathrm{kWh})$, em relação ao pico de consumo do período de aulas da manhã.

Nos dias 08 e 09 de novembro de 2014, ocorreu o Exame Nacional do Ensino Médio (ENEM), tal que as salas de aula monitoradas pela medição foram utilizadas para a aplicação das provas. Portanto, os dados obtidos nestes dois dias foram descartados, pois estes dados não correspondem ao perfil típico de utilização das salas de aula monitoradas.

Figura 5 - Energia Ativa Consumida, em kWh, Medida Durante uma Semana em 5 Salas de Aula da Faculdade de Medicina.

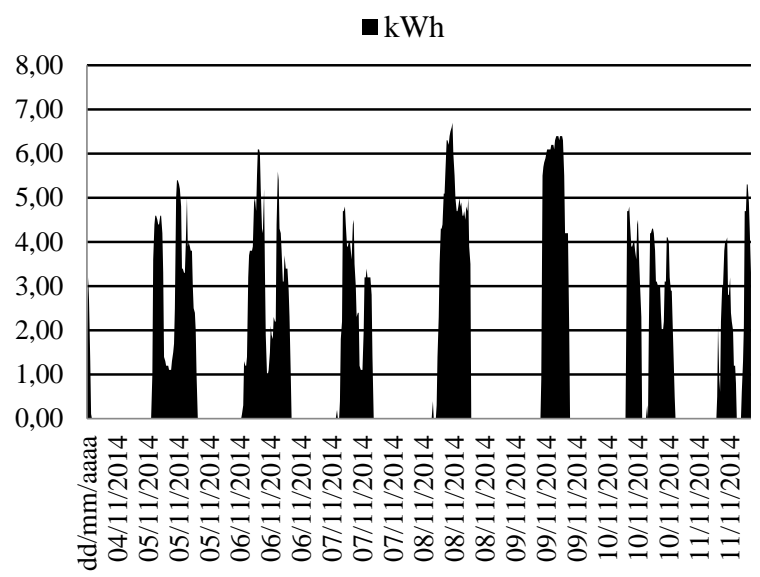

Fonte: Autoria Própria (2015).

A título de exemplo, é mostrado na Figura 6 o consumo de energia elétrica ativa, em $\mathrm{kWh}$, medido das 5 salas de aula (estudo de caso no qual se baseia este trabalho) no dia $06 / 11 / 2014$, o qual se assemelha ao comportamento da demanda, exceto pelo período da noite até antes do início das aulas da manhã (22h00min até 07h30min), de um dia típico de atividades no câmpus da UFMT em Cuiabá, mostrado na Figura 4. Verifica-se, portanto, que a curva da demanda da UFMT é fortemente influenciada pelo uso dos condicionadores de ar.

Figura 6 - Energia Ativa Consumida, em kWh, Medida no dia 06/11/2014 em 5 Salas de Aula da Faculdade de Medicina.

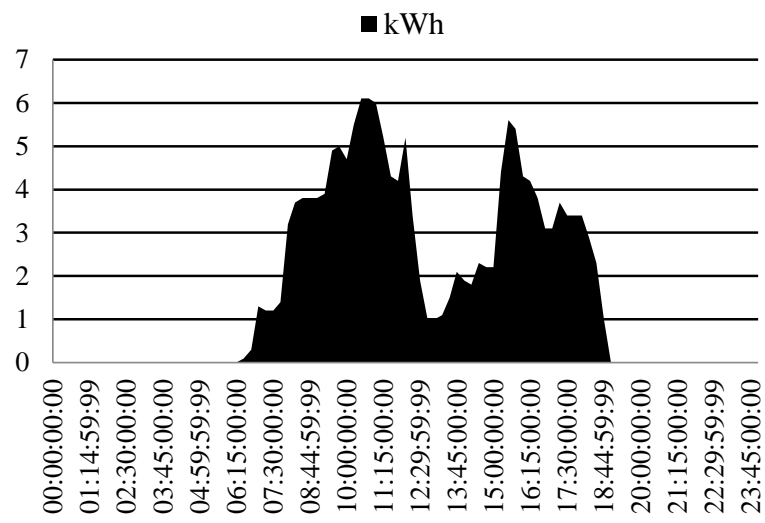

Fonte: Autoria Própria (2015).

A Tabela 2 resume o resultado das medições para o referido período analisado, 
explicitando o consumo de energia elétrica em cada período do dia.

Tabela 2 - Consumo de energia elétrica por período, medido de 4 à 11 de novembro de 2014.

\begin{tabular}{|c|c|c|}
\hline Período & $\begin{array}{l}\text { Horário de } \\
\text { aula (kWh) }\end{array}$ & $\begin{array}{lr}\text { Fora do } \\
\text { horário de } \\
\text { aula }(\mathrm{kWh})\end{array}$ \\
\hline $07: 25$ às $11: 30$ & 294,40 & 1 \\
\hline $11: 31$ às $13: 24$ & 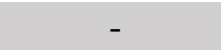 & 45,40 \\
\hline $13: 25$ às $17: 30$ & 250,90 & - \\
\hline $17: 31$ às $17: 54$ & - & 14,30 \\
\hline $17: 55$ às $22: 00$ & 45,60 & - \\
\hline $22: 01$ às $07: 24$ & - & 10,60 \\
\hline Total Parcial & 590,90 & 70,30 \\
\hline Total Geral & \multicolumn{2}{|c|}{661,20} \\
\hline
\end{tabular}

Fonte: Autoria Própria (2015).

Verifica-se, pela Tabela 2, que o consumo de energia elétrica fora do horário previsto para atividades dentro das salas de aula é de 70,30 kWh, o que representa $11,9 \%$ do montante de energia medido durante uma semana no horário de aulas.

\section{Cálculo da Economia de Energia Elétrica}

A UFMT pertence ao subgrupo A4 (2,3 $\mathrm{kV}$ a $25 \mathrm{kV}$ ) com Tarifa Horária Verde. A Tarifa de Energia (TE) no horário de ponta, definido pela Energisa Mato Grosso como o período do dia que vai das $19 \mathrm{~h} 00 \mathrm{~min}$ até as $22 \mathrm{~h} 00 \mathrm{~min}$, é de $\mathrm{R} \$ / \mathrm{kWh} 1,23289$ e para as demais horas do dia, fora de ponta, é de $\mathrm{R} \$ / \mathrm{kWh}$ 0,29898 (ANEEL, 2015a). Estes valores de tarifa não consideram impostos.

O valor do ICMS é de $27,00 \%$, o valor do COFINS é de $0,77 \%$ e do PIS é de $0,16 \%$ (exceto pelo ICMS, os valores de PIS e COFINS variam a cada mês, porém, a título de exemplo, estes valores foram retirados da fatura de energia elétrica da UFMT do mês de fevereiro de 2015).

A bandeira tarifária vigente em todos os subsistemas do Brasil, no ano de 2015, foi a vermelha (ANEEL, 2015b). Portanto, para cada $\mathrm{kWh}$ consumidos a tarifa sofre acréscimo de $\mathrm{R} \$$ 0,045, então, com 70,30 kWh consumidos fora dos horários de aula, o valor final terá acréscimo de $\mathrm{R} \$ 3,16$. Portanto, o valor em Reais $(R \$)$ energia elétrica consumida fora dos horários de aula, durante uma semana, é dado pela expressão abaixo:

$\mathrm{R} \$=\left(\right.$ Adic. $\left._{\text {Band. Vermelha }}\right)+\left\{\left[\left(\right.\right.\right.$ Consumo $_{\text {PONTA }} \times$ TE $\left._{\text {PONTA }}\right)+\left(\right.$ Consumo $_{\text {FORA DE PONTA }} \times$ TE $\left.\left.\left._{\text {FORA DE PONTA }}\right)\right] \mathrm{x}\left[\frac{1}{1-(\text { ICMS+PIS+COFINS })}\right]\right\}$

Onde:

Adic. $\cdot$ Band.Vermelha $=$ Adicional de Bandeira Vermelha $(\mathrm{R} \$)$.

Consumo $_{\text {Ponta }}=$ Consumo de Energia Elétrica no Posto Tarifário PONTA $(\mathrm{kWh})$.

TE $_{\text {PONTA }}=$ Tarifa de Energia Elétrica no Posto Tarifário PONTA (R \$/kWh).

Consumo $_{\text {FORA DE PONTA }}=$ Consumo de Energia Elétrica no Posto Tarifário FORA DE PONTA (kWh).
TE $_{\text {FORA DE PONTA }}=$ Tarifa de Energia Elétrica no Posto Tarifário FORA DE PONTA $(\mathrm{R} \$ / \mathrm{kWh})$.

ICMS = Imposto sobre Circulação de Mercadorias e Serviços.

PIS= Programas de Integração Social

COFINS = Contribuição para Financiamento da Seguridade Social.

Substituindo os valores tem-se:

$$
\mathrm{R} \$=(3,16)+\left\{[(0 \times 1,23289)+(70,30 \times 0,29898)] \mathrm{x}\left[\frac{1}{1-(0,27+0,0016+0,0077)}\right]\right\}=32,32
$$

Como esta quantidade de energia pode-se prever que, em um mês, a quantidade elétrica foi medida durante uma semana, de energia elétrica consumida fora dos 
horários de aula, nas 5 salas de aula monitoradas, será 4 vezes maior, o que resulta em R\$129,28.

\section{Análise das Estratégias para o Controle dos Condicionadores de Ar}

\section{Análise da Primeira Estratégia}

A primeira estratégia de funcionamento para o sistema de controle utiliza um Interruptor Horário, programado para permitir a utilização dos condicionadores de ar no horário das aulas, ou seja: das 07h25min às $11 \mathrm{~h} 30 \mathrm{~min}$, das $13 \mathrm{~h} 25 \mathrm{~min}$ às $17 \mathrm{~h} 30 \mathrm{~min}$ e das $17 \mathrm{~h} 5 \mathrm{~min}$ às $22 \mathrm{~h} 00 \mathrm{~min}$. Esta configuração teve por objetivo evitar o uso dos aparelhos fora dos horários previstos para a realização de atividades dentro das salas de aula, tais como: horário de almoço (entre 11h31min e 13h24min), intervalo entre as aulas (das $17 \mathrm{~h} 31 \mathrm{~min}$ até as $17 \mathrm{~h} 54 \mathrm{~min})$ e das $22 \mathrm{~h} 01 \mathrm{~min}$ às $07 \mathrm{~h} 24 \mathrm{~min}$. De acordo com os resultados presentes na Tabela 2, o consumo medido fora dos horários previstos para a realização de atividades dentro das salas de aula representa $11,9 \%$ do total de energia elétrica, portanto, a princípio, esta é a economia de energia elétrica alcançada, com a implantação da primeira estratégia. $\mathrm{Na}$ Tabela 3 , estão listados os materiais necessários para a implantação da primeira estratégia, mediante o cenário de um conjunto de 5 salas de aula, onde existem os mesmos condicionadores de ar listados na Tabela 1.

A capacidade de condução de corrente elétrica, em regime permanente, dos contatos de força da contatora escolhida, foi determinada de acordo com a medição realizada, onde verificou-se que a corrente elétrica atingiu picos de 115 A. Portanto, o valor comercial superior mais próximo é de 150 A. Contudo, para a elaboração de preço, e por Sinapi (2015) ser utilizada pelo setor de compras da UFMT para aquisição de materiais, foi considerada uma contatora de 185 A, sendo este o valor mais próximo encontrado. Os valores contidos na Tabela 3 foram retirados de Sinapi (2015), com exceção do Interruptor Horário (PLANETA FRIO, 2015). O preço unitário dos cabos, informado na Tabela 3 , se refere ao preço por metro do referido item.

Tabela 3 - Relação de Materiais e Custos da Primeira Estratégia, para o Cenário com 5 Salas de Aula.

\begin{tabular}{|c|c|c|c|}
\hline Descrição & Unidade & Qtde & $\begin{array}{c}\text { Preço } \\
\text { unitário }(\mathrm{R} \$)\end{array}$ \\
\hline Contatora $185 \mathrm{~A}$ & Unidade & 01 & 1640,48 \\
\hline Interruptor horário & Unidade & 01 & 119,35 \\
\hline $\begin{array}{l}\text { Cabo de cobre isolado PVC } 450 / 750 \text { v } 35 \mathrm{~mm}^{2} \text { resistente à } \\
\text { chama }\end{array}$ & Metro & 08 & 13,43 \\
\hline $\begin{array}{l}\text { Cabo de cobre isolado PVC } 450 / 750 \text { v } 2,5 \mathrm{~mm}^{2} \text { resistente à } \\
\text { chama }\end{array}$ & Metro & 05 & 1,47 \\
\hline Disjuntor termomagnético din bipolar 10 a & Unidade & 01 & 34,30 \\
\hline Conectores para cabo $35 \mathrm{~mm}^{2}$ & Unidade & 03 & 3,41 \\
\hline $\begin{array}{l}\text { Quadro de distribuição de sobrepor, em chapa metálica, } \\
\text { com medidas } 50 \mathrm{~cm} \times 50 \mathrm{~cm}\end{array}$ & Unidade & 01 & 929,37 \\
\hline PREÇO TOTAL $(\mathbf{R} \$)$ & & & 2848,52 \\
\hline
\end{tabular}

Fonte: Autoria Própria (2015), a partir de Sinapi (2015) e Planeta Frio (2015).

O modelo mais comum de Interruptor Horário é do tipo digital, com uma ou duas saídas a relé, onde o modelo com uma saída possui um relé de $16 \mathrm{~A} / 250 \mathrm{~V}_{\mathrm{CA}}$ e o de duas saídas possui dois relés de $8 \mathrm{~A} / 250 \mathrm{~V}_{\mathrm{CA}}$. A bobina de uma contatora de 185 A consome aproximadamente $0,65 \mathrm{~A}$, quando energizada (WEG, 2015). Logo, um Interruptor Horário, 
com uma ou duas saídas, pode alimentar, simultaneamente, as bobinas de até 24 contatoras de potência, onde cada contatora é responsável por acionar, simultaneamente, os condicionadores de ar de 05 salas de aula, o que resulta em 120 salas de aula comandadas por apenas um Interruptor Horário.

\section{Análise da Segunda Estratégia}

A segunda estratégia de funcionamento do sistema de controle utiliza um CLP operando da mesma forma que um Interruptor Horário, ou seja, permitindo a utilização dos aparelhos nos mesmos horários e com os mesmos objetivos da primeira estratégia. Logo, o potencial de economia de energia elétrica da segunda estratégia também é de 11,9\%. Na Tabela 4 estão listados os materiais necessários à implantação da segunda estratégia, mediante o cenário de um conjunto de 5 salas de aula, onde existem os mesmos condicionadores de ar listados na Tabela 1 . a relé, onde o CLP especificado possui 8 saídas, enquanto o Interruptor Horário possui uma ou duas saídas. E mais, cada saída do CLP possui um relé de $8 \mathrm{~A} / 250 \mathrm{~V}_{\text {CA. Portanto, }}$ analisando pela ótica da potência das cargas a serem acionadas nas saídas dos dispositivos, utilizar um CLP nesta concepção é igual a utilizar 04 Interruptores Horários.

Como explicado na análise da primeira estratégia, a contatora utilizada para a segunda estratégia também é de $185 \mathrm{~A}$.

Os valores contidos na Tabela 4 foram retirados de Sinapi (2015), com exceção do CLP (ALI EXPRESS, 2015). O preço unitário dos cabos, informado na Tabela 4 , se refere ao preço por metro do referido item.

A diferença entre o CLP e o Interruptor Horário especificados é a quantidade de saídas

Tabela 4 - Relação de Materiais e Custos da Segunda Estratégia, para o Cenário com 5 Salas de Aula.

\begin{tabular}{|c|c|c|c|}
\hline Descrição & Unidade & Qtde & $\begin{array}{c}\text { Preço } \\
\text { unitário }(\mathrm{R} \$)\end{array}$ \\
\hline Contatora $185 \mathrm{~A}$ & Unidade & 01 & 1640,48 \\
\hline $\mathrm{Clp}$ & Unidade & 01 & $745,00^{1}$ \\
\hline $\begin{array}{l}\text { Cabo de cobre isolado PVC } 450 / 750 \text { v } 35 \mathrm{~mm}^{2} \text { resistente à } \\
\text { chama }\end{array}$ & Metro & 08 & 13,43 \\
\hline $\begin{array}{l}\text { Cabo de cobre isolado PVC } 450 / 750 \text { v } 2,5 \text { mm}^{2} \text { resistente à } \\
\text { chama }\end{array}$ & Metro & 05 & 1,47 \\
\hline Disjuntor termomagnético din bipolar 10 a & Unidade & 01 & 34,30 \\
\hline Conectores para cabo $35 \mathrm{~mm}^{2}$ & Unidade & 03 & 3,41 \\
\hline $\begin{array}{l}\text { Quadro de distribuição de sobrepor, em chapa metálica, } \\
\text { com medidas } 50 \mathrm{~cm} \times 50 \mathrm{~cm}\end{array}$ & Unidade & 01 & 929,37 \\
\hline PREÇO TOTAL (R\$) & & & 3474,17 \\
\hline
\end{tabular}

Fonte: Autoria Própria (2015), a partir de Sinapi (2015) e Ali Express (2015).

${ }^{1}$ Cotação do dólar no dia 21 de maio de 2015: R\$ 3,0411. 
A utilização do CLP resulta na possibilidade de acionamento simultâneo de até 96 bobinas de contatoras de potência, onde a contatora considerada nesta análise é a mesma da primeira estratégia, ou seja, sua bobina consome aproximadamente $0,65 \mathrm{~A}$, quando energizada (WEG, 2015), tal que cada contatora é responsável por acionar, simultaneamente, os condicionadores de ar de 05 salas de aula, o que resulta em 480 salas de aula comandadas por apenas um CLP.

\section{DISCUSSÕES}

A UFMT possui muitos blocos de salas de aula antigos, construídos há quase 40 anos, onde as instalações elétricas foram projetadas para que fossem utilizados ventiladores de teto dentro das salas de aula. Portanto, a atual configuração das instalações elétricas possui poucos quadros de distribuição com circuitos exclusivos de condicionadores de ar. Logo, é necessário realizar adequações nas instalações elétricas, para que existam mais quadros de distribuição exclusivos para condicionadores de ar, para que o sistema de controle possa ser reproduzido.

A primeira estratégia de funcionamento do sistema de controle dos condicionadores de ar permite que até 120 salas de aula sejam comandadas por apenas um Interruptor Horário, com uma economia de energia elétrica da ordem de $11,9 \%$ do total de energia elétrica consumida, desde que o perfil de consumo das 120 salas de aula seja semelhante ao das 5 salas de aula monitoradas. Percebe-se que esta concepção do sistema de controle permite que apenas um Interruptor Horário seja responsável pelo acionamento de todos os condicionadores de ar, de um bloco típico de salas de aulas da UFMT, desde que seja utilizada uma contatora para cada quadro de distribuição. Como consequência desta característica, o maior risco oferecido com o uso deste arranjo é o de empregar apenas um dispositivo de controle para a utilização de muitos aparelhos. Portanto, com a saída de operação ou uma falha do sistema, como no caso de uma manutenção ou de um componente danificado, muitas salas de aula seriam atingidas. Outra consideração importante é que esta concepção não possibilita a configuração online dos Interruptores Horários.Ou seja, é necessário ir até o local onde se encontra o sistema de controle para programar o Interruptor Horário.

Considerando que a economia de energia elétrica alcançada por esta estratégia, para o cenário que considera 5 salas de aula, representa $\mathrm{R} \$ 129,28$ por mês e, para o mesmo cenário, o seu custo de implantação, é de R \$ 2848,52 , então o seu tempo de retorno do investimento é de 22,03 meses (esta é uma estimativa simples, que não considera os detalhes da teoria econômica, como taxa de juros, por exemplo). A vida útil média dos equipamentos utilizados neste arranjo vai de 2 a $15 \operatorname{anos}$ ( 24 a 180 meses).

A seguir, são apresentados alguns cenários, com 10, 15 e 20 salas de aula, para a implantação da primeira estratégia, considerando que o perfil de consumo para cada cenário seja semelhante à medição realizada em 5 salas de aula. Entende-se que, além do risco de utilizar apenas um dispositivo para controlar muitos aparelhos, como mencionado anteriormente, nenhum bloco da UFMT possui 120 salas de aula. Portanto, torna-se tecnicamente inviável empregar apenas um dispositivo para controle do sistema em um número elevado de salas de aula. No outro extremo, em um cenário onde seriam utilizados um Interruptor Horário para controlar cada sala de aula, totalizando 120 Interruptores Horários, o projeto apresentaria investimento inicial elevado, com consequente inviabilidade econômica.

Para a composição do valor do investimento inicial de cada cenário da primeira estratégia, considerou-se como referência a Tabela 3. Portanto, foram feitas as seguintes considerações:

- Uma contatora de potência, de 185 A, para cada conjunto de 5 salas de aula; 
- Um Interruptor Horário, pois pode controlar até 120 salas;

- Os cabos de $35 \mathrm{~mm}^{2}$ e $2,5 \mathrm{~mm}^{2}$ tiveram suas quantidades dobradas para cada 5 salas acrescentadas;

- 3 (três) conectores para cabo $35 \mathrm{~mm}^{2}$ para cada contatora de potência;

- Um disjuntor termomagnético e um quadro de distribuição para cada Interruptor Horário.

Nesta análise considerou-se que havia espaço suficiente para alocar o contator nos quadros de distribuição de cada cenário, não sendo necessário utilizar mais quadros de distribuição. Desta forma, foi elaborada a Tabela 5, a qual resume os resultados obtidos para cada cenário da primeira estratégia.

Tabela 5 - Resumo dos Resultados Obtidos para cada Cenário da Primeira Estratégia.

\begin{tabular}{cccc}
\hline $\begin{array}{c}\text { Cenário } \\
\text { (salas) }\end{array}$ & $\begin{array}{c}\text { Investimento } \\
(\mathrm{R} \$)\end{array}$ & $\begin{array}{c}\text { Economia } \\
\text { mensal } \\
(\mathrm{R} \$)\end{array}$ & $\begin{array}{c}\text { Tempo de } \\
\text { Retorno } \\
\text { (meses) }\end{array}$ \\
\hline 5 & 2848,52 & 129,28 & 22,03 \\
10 & 4614,02 & 258,56 & 17,85 \\
15 & 6379,52 & 387,84 & 16,45 \\
20 & 8145,02 & 517,12 & 15,75 \\
\hline
\end{tabular}

Fonte: Autoria Própria (2015).

Agora, considerando-se que não há espaço suficiente no quadro de distribuição para alocar a contatora de potência em cada cenário, foi adicionado um quadro de distribuição de sobrepor, em chapa metálica, com medidas $30 \mathrm{~cm}$ x $30 \mathrm{~cm}$, com preço de $\mathrm{R} \$$ 250,32 (SINAPI, 2015) para cada conjunto de 5 salas de aula, exceto para as primeiras 5 salas. Desta forma, foi elaborada a Tabela 6, a qual resume os resultados obtidos para cada cenário da primeira estratégia com o quadro de distribuição adicional para cada conjunto de 5 salas de aula.
Tabela 6 - Resumo dos Resultados Obtidos para cada Cenário da Primeira Estratégia com Quadros de Distribuição amais.

\begin{tabular}{cccc}
\hline $\begin{array}{c}\text { Cenário } \\
\text { (salas) }\end{array}$ & $\begin{array}{c}\text { Investimento } \\
(\mathrm{R} \$)\end{array}$ & $\begin{array}{c}\text { Economia } \\
\text { mensal } \\
(\mathrm{R} \$)\end{array}$ & $\begin{array}{c}\text { Tempo de } \\
\text { Retorno } \\
(\text { meses })\end{array}$ \\
\hline 5 & 2848,52 & 129,28 & 22,03 \\
10 & 4864,34 & 258,56 & 18,81 \\
15 & 6880,16 & 387,84 & 17,74 \\
20 & 8895,98 & 517,12 & 17,20 \\
\hline
\end{tabular}

Fonte: Autoria Própria (2015).

A segunda estratégia apresenta custo de aquisição dos equipamentos, do cenário apresentado, maior do que a primeira estratégia, permitindo que até 480 salas de aula sejam comandadas por apenas um CLP, com uma economia de energia elétrica da ordem de $11,9 \%$ do total de energia elétrica consumida, desde que o perfil de consumo das 480 salas seja semelhante ao das 5 salas de aula monitoradas. Da mesma forma que na primeira estratégia, o maior risco oferecido com o uso deste arranjo é o de empregar apenas um dispositivo de controle para a utilização de muitos aparelhos. Portanto, com a saída de operação ou uma falha do sistema, como no caso de uma manutenção ou de um componente danificado, muitas salas de aula seriam atingidas. É necessário ressaltar que a equipe de manutenção das instalações elétricas da UFMT não possui profissionais habilitados para realizarem a programação ou a manutenção de um CLP. Logo, é preciso que a equipe responsável pela manutenção do sistema de controle realize curso de capacitação para a operação e manutenção deste equipamento. Também é importante citar que a utilização do CLP possibilita empregar sistemas supervisórios, para $\mathrm{o}$ monitoramento à distância e em tempo real do controle dos condicionadores de ar. Isto significa que o sistema de controle pode ser configurado online, onde, por exemplo, todos os CLP's de um bloco de salas de aula, ou de vários blocos, podem estar disponíveis para configuração de sua programação com a utilização de apenas um computador, o que se assemelha à criação de uma 'Central de 
Controle de Acionamento de Condicionadores de Ar', que pode ser expandida para todos os blocos de salas de aula da universidade.

Considerando que a economia de energia elétrica alcançada por esta estratégia, para o cenário que considera 5 salas de aula, representa $\mathrm{R} \$ 129,28$ por mês e, para o mesmo cenário, o seu custo de implantação, é de $\mathrm{R} \$$ 3474,17 , então o seu tempo de retorno do investimento é de 26,87 meses (esta é uma estimativa simples que não considera os detalhes da teoria econômica, como taxa de juros, por exemplo). A vida útil média dos equipamentos utilizados neste arranjo vai de 2 anos a 15 anos (24 a 180 meses).

Outra possibilidade oferecida pela segunda estratégia utiliza uma saída do CLP para cada sala de aula, considerando o cronograma de ocupação das salas de aula (horário das aulas). Logo, cada saída do CLP deve possuir uma programação diferente, necessitando de uma contatora de potência, responsável pelo acionamento/desligamento dos condicionadores de ar nos horários de ocupação das salas, para cada saída utilizada. Este arranjo, proveniente da segunda estratégia, necessita de mais equipamentos para ser implantado, pois, para cada sala de aula, é necessário utilizar uma contatora de potência. Logo, seu custo de implantação é mais elevado do que a primeira e segunda estratégias, somada à necessidade de se ter um estoque maior de equipamentos do sistema de controle, para eventuais trocas em manutenções. Para este arranjo, a saída de operação ou uma falha do sistema, como no caso de uma manutenção, de uma contatora ou de um conjunto de CLP's de determinado bloco de salas de aula, não ocasiona, necessariamente, o desligamento de todo o sistema de controle, o que pode ser considerado interessante do ponto de vista operacional.

Da mesma forma que a segunda estratégia, também é possível empregar sistemas supervisórios para o monitoramento à distância e em tempo real do controle dos condicionadores de ar. Além disso, existe a possibilidade de utilizar outros dispositivos como sensores, para auxiliar a tomada de decisões do CLP e permitir que seja realizado um controle mais preciso da utilização dos condicionadores de ar, como sensores de presença e sensores de temperatura.

A seguir, são apresentados alguns cenários, com 10, 15 e 20 salas de aula, para a implantação da segunda estratégia, considerando que o perfil de consumo para cada cenário seja semelhante à medição realizada em 5 salas de aula. Entende-se que, além do risco de utilizar apenas um dispositivo para controlar muitos aparelhos, como mencionado anteriormente, nenhum bloco da UFMT possui 480 salas de aula. Portanto, torna-se tecnicamente inviável empregar apenas um dispositivo para controle do sistema em um número elevado de salas de aula. No outro extremo, em um cenário onde seriam utilizados um CLP para controlar cada sala de aula, totalizando 480 CLP's, o projeto apresentaria um investimento inicial elevado, com consequente inviabilidade econômica.

Para a composição do valor do investimento inicial de cada cenário da segunda estratégia, considerou-se como referência a Tabela 4. Portanto, foram feitas as seguintes considerações:

- Uma contatora de potência, de 185 A, para cada conjunto de 5 salas de aula;

- Um CLP, pois pode controlar até 480 salas;

- Os cabos de $35 \mathrm{~mm}^{2}$ e $2,5 \mathrm{~mm}^{2}$ tiveram suas quantidades dobradas para cada 5 salas acrescentadas;

- 3 (três) conectores para cabo $35 \mathrm{~mm}^{2}$ para cada contatora de potência;

- Um disjuntor termomagnético e um quadro de distribuição para cada CLP.

Nesta análise considerou-se que havia espaço suficiente para alocar o contator nos quadros de distribuição de cada cenário, não sendo necessário utilizar mais quadros de distribuição. Desta forma, foi elaborada a 
Menegotto et.al., Análise de Duas Tecnologias Comercialmente Disponíveis para o Controle do Sistema de Condicionamento de Ar da UFMT: Um Estudo de Caso. E\&S - Engineering and Science, (2016), 5:1.

Tabela 7, a qual resume os resultados obtidos para cada cenário da segunda estratégia.

Tabela 7 - Resumo dos Resultados Obtidos para cada Cenário da Segunda Estratégia.

\begin{tabular}{cccc}
\hline $\begin{array}{c}\text { Cenário } \\
\text { (salas) }\end{array}$ & $\begin{array}{c}\text { Investimento } \\
(\mathrm{R} \$)\end{array}$ & $\begin{array}{c}\text { Economia } \\
\text { mensal } \\
(\mathrm{R} \$)\end{array}$ & $\begin{array}{c}\text { Tempo de } \\
\text { Retorno } \\
\text { (meses) }\end{array}$ \\
\hline 5 & 3474,17 & 129,28 & 26,87 \\
10 & 5239,67 & 258,56 & 20,26 \\
15 & 7005,17 & 387,84 & 18,06 \\
20 & 8770,67 & 517,12 & 16,96 \\
\hline
\end{tabular}

Fonte: Autoria Própria (2015).

Agora, considerando-se que não há espaço suficiente no quadro de distribuição para alocar a contatora de potência em cada cenário, foi adicionado um quadro de distribuição de sobrepor, em chapa metálica, com medidas $30 \mathrm{~cm}$ x $30 \mathrm{~cm}$, com preço de $\mathrm{R} \$$ 250,32 (SINAPI, 2015) para cada conjunto de 5 salas de aula, exceto para as primeiras 5 salas. Desta forma, foi elaborada a Tabela 8, a qual resume os resultados obtidos para cada cenário da segunda estratégia com o quadro de distribuição adicional para cada conjunto de 5 salas de aula.

Tabela 8 - Resumo dos Resultados Obtidos para cada Cenário da Segunda Estratégia com Quadros de Distribuição amais.

\begin{tabular}{cccc}
\hline $\begin{array}{c}\text { Cenário } \\
\text { (salas) }\end{array}$ & $\begin{array}{c}\text { Investimento } \\
(\mathrm{R} \$)\end{array}$ & $\begin{array}{c}\text { Economia } \\
\text { mensal } \\
(\mathrm{R} \$)\end{array}$ & $\begin{array}{c}\text { Tempo de } \\
\text { Retorno } \\
\text { (meses) }\end{array}$ \\
\hline 5 & 3474,17 & 129,28 & 26,87 \\
10 & 5490,00 & 258,56 & 21,23 \\
15 & 7505,81 & 387,84 & 19,35 \\
20 & 9521,63 & 517,12 & 18,41 \\
\hline
\end{tabular}

Fonte: Autoria Própria (2015).

A Figura 7 mostra os tempos de retorno, em meses, para cada cenário analisado, da primeira e segunda estratégias, sem a adição de quadros de distribuição.
Figura 7 - Tempo de Retorno, em Meses, para cada Cenário Analisado, da Primeira e Segunda Estratégias, sem a Adição de Quadros de Distribuição.

Tempo de Retorno

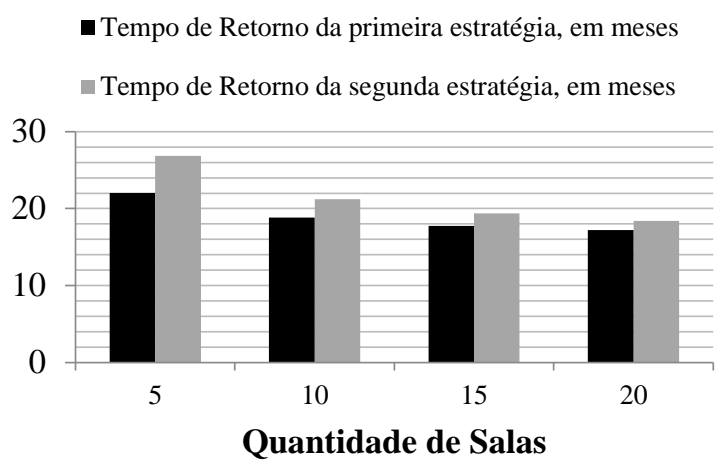

Fonte: Autoria Própria (2015).

A Figura 8 mostra os tempos de retorno, em meses, para cada cenário analisado, da primeira e segunda estratégias, com a adição de quadros de distribuição.

Figura 8 - Tempo de Retorno, em Meses, para cada Cenário Analisado, da Primeira e Segunda Estratégias, com a Adição de Quadros de Distribuição.

\section{Tempo de Retorno}

- Tempo de Retorno da primeira estratégia, em meses

- Tempo de Retorno da segunda estratégia, em meses

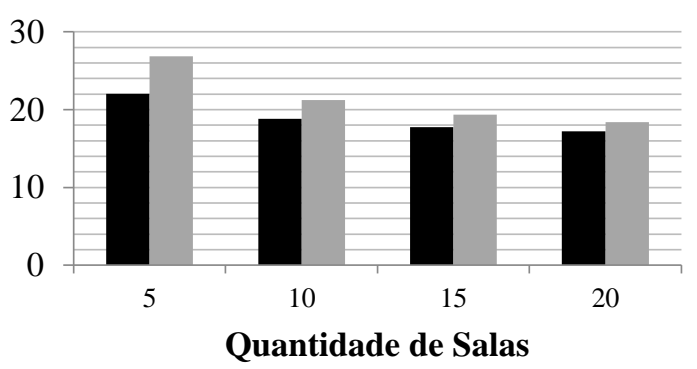

Fonte: Autoria Própria (2015).

\section{CONCLUSÕES}

As duas estratégias de funcionamento do sistema de controle atingem o objetivo de reduzir o consumo de energia elétrica com baixo investimento inicial, visto que hoje a UFMT não possui um sistema de controle para os condicionadores de ar. Porém, a adoção, de uma determinada estratégia, por 
parte das áreas técnicas na UFMT, dependerá do planejamento financeiro e de investimentos da Universidade, envolvendo a disponibilidade de recursos para investimento inicial, análise do tempo de retorno do investimento, o planejamento da manutenção do sistema, a quantidade de peças em estoque, a quantidade de salas atendidas pelo sistema, treinamento da equipe responsável, entre outros.

Neste trabalho foi realizada uma análise teórica acerca de duas tecnologias comercialmente disponíveis para o controle do sistema de condicionamento de ar da UFMT, onde observa-se que é possível realizar diversos tipos de controles, relacionados à utilização de condicionadores de ar, como controle do tempo de utilização, temperatura interna dos ambientes, acionamento/desligamento à distância, entre outros, onde estas variáveis ocasionam um impacto direto no consumo de energia elétrica em sistemas de ar condicionado. Para tanto, foram analisadas duas estratégias de funcionamento do sistema de controle, uma utilizando um Interruptor Horário e outra utilizando um CLP.

Os ganhos obtidos com a primeira estratégia estão na possibilidade de acionar um bloco inteiro de salas de aula, com apenas um Interruptor Horário e o baixo custo inicial para a aquisição dos equipamentos, com tempo de retorno de 22,03 meses, para o cenário com 5 salas de aula, onde o maior risco desta concepção reside no fato de que a saída de operação do sistema pode atingir uma grande quantidade de salas de aula. Esta estratégia é mais simples, em relação à segunda, do ponto de vista operacional, pois é mais fácil realizar a programação de um Interruptor Horário do que de um CLP, o que acarreta ganhos significativos.

Foram analisados alguns cenários para a primeira estratégia, onde se percebe uma redução no tempo de retorno do investimento, com o aumento da quantidade de salas de aula abrangidas pelo sistema de controle. Desta forma, ressalta-se a viabilidade financeira desta estratégia.

Os ganhos obtidos com a segunda estratégia estão na possibilidade de acionar vários blocos de salas de aula próximos com apenas um CLP, além de possuir uma abrangência maior do que a primeira estratégia e a possibilidade de expansão, através do emprego de sistemas supervisórios online para o monitoramento à distância e em tempo real do sistema de controle, com tempo de retorno de 26,87 meses para o cenário com 5 salas de aula, onde, do mesmo modo que na primeira estratégia, o maior risco desta concepção reside no fato de que a saída de operação do sistema atinge uma grande quantidade de salas de aula. Com esta estratégia vislumbra-se a possibilidade de expansão do sistema para o controle individual de cada sala de aula, permitindo a utilização de dispositivos do tipo sensores, para incrementar o controle do uso dos condicionadores de ar.

Portanto, deve-se analisar a quantidade de salas de aula a serem atendidas pelo sistema de controle, para identificar a estratégia mais adequada, levando-se em consideração o custo inicial de implantação, a vida útil dos equipamentos e o potencial de economia de energia elétrica obtido.

Também foram analisados alguns cenários para a segunda estratégia, onde percebe-se uma redução no tempo de retorno do investimento, com o aumento da quantidade de salas de aula abrangidas pelo sistema de controle. Desta forma, ressalta-se a viabilidade financeira desta estratégia.

Existem outras tecnologias que permitem criar sistemas de controle de condicionadores de ar, porém, as mais comuns, são as tratadas neste trabalho. Um exemplo de outra tecnologia recente são os microcontroladores que utilizam a tecnologia Arduino. 


\section{REFERÊNCIAS}

Ali

Express 2015.

$<$ http://pt.aliexpress.com/item/Schneider-12basic-logic-controllerinput-relay-output-at-8-oclock-SR2E201FU/1877854599.html> Acesso em: 21 maio 2015.

ANEEL - Agência Nacional de Energia Elétrica. 2015a. Resolução Homologatória no 1.873, de 7 de Abril de 2015. Disponível em:<http://www.aneel.gov.br/cedoc/reh2015187 3ti.pdf> Acesso em: 25 jun. 2015.

ANEEL - Agência Nacional de Energia Elétrica. 2015b. Relatório do Acionamento das Bandeiras Tarifárias. Disponível em: $<$ http://www.aneel.gov.br/arquivos/PDF/Relatori o_do_Acionamento_das_Bandeiras_

Tarifarias_jul2015.pdf> Acesso em: 27 jul. 2015.

Planeta Frio 2015 . $<$ http://www.planetafrio.com.br/interruptorhorario-digital-coel-bwt40hr-100-a-240vca-16a48-a-63-hz-cod-11251000-ref-692-34-p999/> Acesso em: 21 maio 2015.

PROCEL - Programa Nacional de Conservação de Energia Elétrica. Avaliação do Mercado de Eficiência Energética do Brasil. Sumário Executivo - Ano Base 2005. 2007. Disponível em: <www.procelinfo.com.br> Acesso em: 25 nov. 2014.

REIS, L. F. F. Análise da Qualidade da Energia Elétrica: Estudo de Caso da Universidade Federal de Mato Grosso (UFMT - Câmpus Cuiabá). Trabalho Final de Curso (Graduação em Engenharia Elétrica). Universidade Federal de Mato Grosso. Cuiabá, 2015.

SANTOS, A. H. M. et al.Conservação de Energia: Eficiência Energética de Equipamentos e Instalações. PROCEL EDUCAÇÃO. Itajubá, 2006.

SINAPI - Sistema Nacional de Pesquisa de Custos e Índices da Construção Civil. Relatório de Insumos de Abril de 2015 para Mato Grosso Publicado em 15 de Maio de 2015. Disponível em: <http://www.caixa.gov.br/Downloads/sinapia-partir-jul-2014-

mt/SINAPI_Preco_Ref_Insumos_MT_042015_D

esonerado.PDF> Acesso em: 21 maio 2015.
WEG. Automação - Catálogo Geral de Contatores e Relés de Sobrecarga. Disponível em: <http://ecatalog.weg.net/files/wegnet/WEGcontatores-e-reles-de-sobrecargacatalogo-geral50026112-catalogo-portugues-br.pdf > Acesso em: 13 abr. 2015. 